DE DE GRUYTER

OPEN

\title{
THE EFFECT OF PLEUROTUS ERYNGII STALK RESIDUE DIETARY SUPPLEMENTATION ON LAYER PERFORMANCE, EGG TRAITS AND OXIDATIVE STATUS*
}

\author{
Tzu-Tai Lee ${ }^{1 \bullet}$, Jhih-Ying $\mathrm{Ciou}^{2}$, Chung-Nan $\mathrm{Chen}^{1}, \mathrm{Bi} \mathrm{Yu}^{1}$ \\ ${ }^{1}$ Department of Animal Science, National Chung Hsing University, Taiwan \\ ${ }^{2}$ Department of Food Science, Tung Hai University, Taiwan \\ •Corresponding author: ttlee@dragon.nchu.edu.tw
}

\begin{abstract}
This study was conducted to investigate the effects of Pleurotus eryngii stalk residue (PESR) on performance, egg traits and oxidative status of laying hens. One hundred and twenty 22-week-old laying hens (Hendrix) were randomly allocated into 4 dietary groups, each containing 30 birds, including 10 replicates per group and 3 birds per replicate. In addition, they were fed diets supplemented with 0 (control group, corn-soybean meal), $0.5,1.0$, or $2.0 \%$ dried PESR groups for 8 weeks, respectively. The results indicated that the different levels of dietary supplementation produced no significant differences in production performance among the experimental groups of laying hens. The Haugh unit was significantly higher in the $1.0 \%$ and $2.0 \%$ PESR groups compared to the control group. The eggs' cholesterol content of the $1.0 \%$ and $2.0 \%$ PESR groups was significantly lower than that of the control group at 5-8 weeks. The blood triglyceride content and cholesterol content were significantly lower in the $1.0 \%$ and $2.0 \%$ PESR groups compared to the control group at the 4th and 8th weeks. Hence, the supplemented $1.0 \%$ and $2.0 \%$ of PESR groups showed higher levels for serum trolox equivalent antioxidant capacity, catalase and superoxide dismutase during the experimental period in comparison with the control group. This study indicated that a by-product of Pleurotus eryngii, PESR, could produce lower-cholesterol eggs and improve the oxidative stability with $1.0 \%$ and $2.0 \%$ PESR supplementation in laying hens.
\end{abstract}

Key words: Pleurotus eryngii stalk residue, laying hens, egg traits, antioxidants, feed additive

Abbreviations used: PESR: Pleurotus eryngii stalk residue; FCR: feed conversion ratio; HPLC: high pressure liquid chromatography; TEAC: trolox equivalent antioxidant capacity; CAT: catalase; SOD: superoxide dismutase; HDL: high-density lipoprotein; DW: dry weight; GAE: gallic acid equivalent

Natural dietary antioxidants are currently receiving considerable attention in animal nutrition fields due to their association with feed high-quality characteristics and their contribution to the protection against oxidant stress. Peroxide is reactive

* The authors thank the Council of Agriculture of Taiwan (100AS-2.1.4-AD-U1) and National Science Council (NSC 101-2313-B-005-050-MY3) for financially supporting this study. 
oxygen species (ROS) that causes oxidative damage, a consequence of insufficient antioxidant potential; excessive oxidative stress is the other possible consequence in animals (Safary and Daneshyar, 2012). Under normal circumstances, the animal could remove excessive ROS using a nonenzymatic antioxidant system and a series of antioxidant enzymes such as catalase (CAT) and superoxide dismutase (SOD) (Seven et al., 2010; Safary and Daneshyar, 2012).

A high antioxidative status has been regarded as one of the major factors positively affecting the production performance in the intensive poultry industry (Lin et al., 2006; Mujahid et al., 2007). In addition, feedstuff can be easily oxidized during processing and storage by a series of oxidative reactions, which would also negatively affect birds' performance (Mujahid et al., 2007). Normally, dietary oxidation stability is one with major factors influencing the shelf life of feed. Therefore, supplementation of the antioxidants to mitigate the oxidative stress has become a commercial practice by the industry in regard to poultry feeding. Today's poultry producers are confronted with numerous challenges to prevent diseases and maintain health without the use of sub-therapeutic antibiotics. As food safety and animal welfare concerns continue to increase, researchers will continue to seek better alternatives to current methods applied to molting laying hens (Lin et al., 2006; Liu et al., 2014). In recent years, there has been increasing interest in the use of natural antioxidants such as wild mushrooms, which are considered good sources of functional ingredients because these natural antioxidants contribute to avoiding undesirable health problems that may arise from the use of synthetic antioxidants such as butylated hydroxyanisole (BHA) and butylated hydroxytoluene (BHT) (Safary and Daneshyar, 2012; Lee et al., 2013). Some researchers have shown that antioxidants appear at high levels in fruits and/or in the mycelia of several mushrooms; as growth and health promoters, they have therefore been utilized in animal diets in Asian countries (Manzi et al., 2004; Mau et al., 2004).

Due to the remarkable flavor and nutritional value of Pleurotus eryngii (P. eryngii, Figure 1A), it has rapidly become a highly valued species among consumers in Asia, Europe and North America. P. eryngii is rich in carbohydrates (approximately $9.6 \%$ of the fresh weight), and a significant number of these carbohydrates are composed of dietary fibers (approximately $4.6 \%$ of fresh weight) and chitin (approximately $0.5 \%$ of fresh weight) (Manzi et al., 1999). Additionally, P. eryngii has abundant potential for producing useful bioactive metabolites, including the phenolic compounds and metabolite biomolecules (such as lovastatin, eryngin, pleureyn, ribonuclease, lectins and 17b-estradiol) (Manzi et al., 1999). Moreover, P. eryngii is also identified as a healthful food because it is lower in lipid/fat (approximately $0.8 \%$ of fresh weight) and calories. Usually, P. eryngii is typically cultivated within an architectural structure inside an air-conditioned structure. When the mature mushrooms are ready to sell, their stalks must be cut and removed as the by-product. Our previous study demonstrated that the in vitro antioxidant activity of the $P$. eryngii does not seriously inhibit the viability of the chickens' peripheral blood mononuclear cells (PBMCs) and suggested that P. eryngii can be used as a potential feed additive (Lee et al., 2011). 
(A)

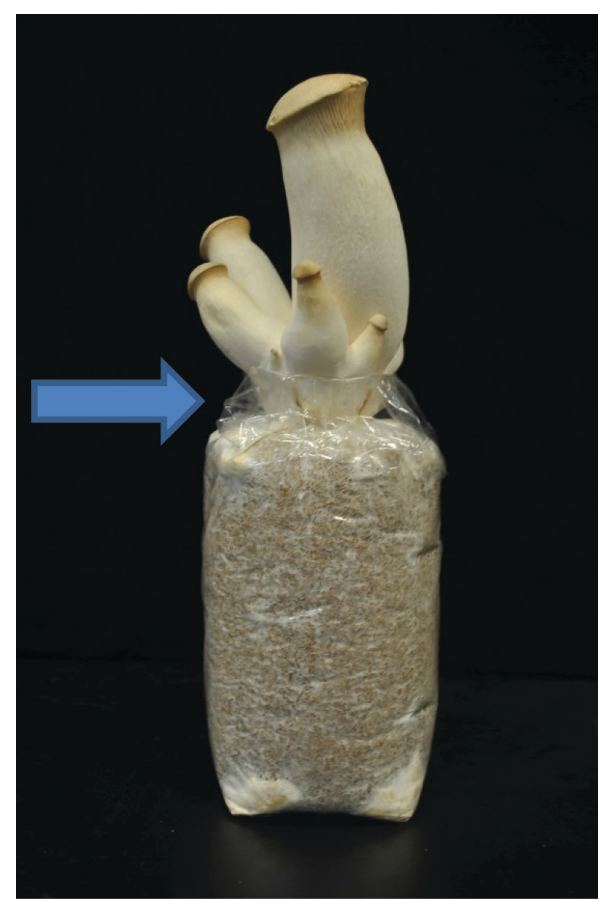

(B)

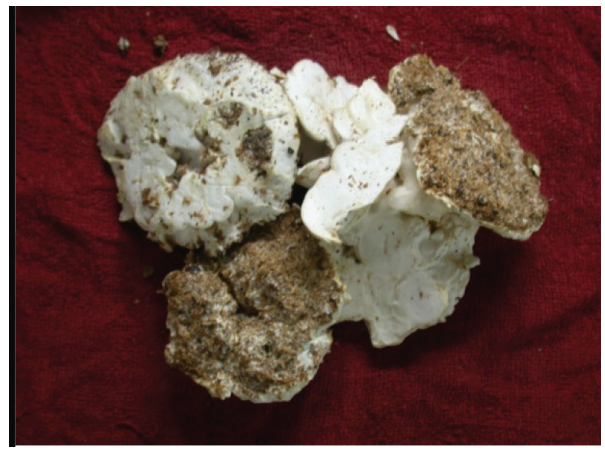

Figure 1. Pleurotus eryngii fruiting body (A) and Pleurotus eryngii stalk residue (B)

To our knowledge, there is little information available on the influence of dietary supplementation with dried Pleurotus eryngii stalk residue (PESR, Figure 1B) powder in hens' diet. Therefore, the objective of the present study was to investigate the effects of dried PESR, a by-product of $P$. eryngii fruiting body, on the laying performance, egg traits and oxidant status. 


\section{Material and methods}

\section{Source and preparation methods for Pleurotus eryngii stalk residue (PESR)}

Pleurotus eryngii stalk residue (PESR) used in this study was kindly provided by a local mushroom producer (Q-YO BIO-TECHNOLOGY Farm, Changhau, Taiwan). The fresh PESR was dried in a forced hot-air dryer at $65^{\circ} \mathrm{C}$ for 3 days, and then ground to a powder (approximately $1 \mathrm{~mm}$ ) prior to being added to the feed. The extracts were added to $100 \%$ distilled water $(1: 10, \mathrm{w} / \mathrm{v})$ at $95^{\circ} \mathrm{C}$ for $1 \mathrm{~h}$ after filtering (Advantec NO. 1, Tokyo, Japan) (Lee et al., 2011). The filtrate was evaporated to dryness under vacuum conditions, and the lyophilized extracts were rehydrated and adjusted to $1 \mathrm{mg} / \mathrm{mL}$ for subsequent analyses. Crude soluble polysaccharide assays were conducted using the phenol-sulfate method. The extracts obtained were analyzed spectrophotometrically by comparing the results to the data from the glucose standard curve at $730 \mathrm{~nm}$. The $\beta$-glucan content was determined by modifying a previously described method (McCleary and Glennie-Holmes, 1985). The total phenolic content was determined using a Folin-Ciocalteu reagent. The content of the phenolic compounds of the extracts was determined as a microgram measurement of the gallic acid equivalent (GAE) by using an equation obtained from a standard gallic acid graph according to the manufacturer's protocols (Kujala et al., 2000). An analysis of the adenosine and ergosterol content by high pressure liquid chromatography (HPLC) was conducted using the Waters Model Alliance 2690 and 996 photodiode array detectors set at wavelengths of 260 and $270 \mathrm{~nm}$ (Waters, Milford, MA, USA), respectively. The separations were achieved with a $15 \mu \mathrm{L}$ loop, a reversedphase column (Merck LiChrospher 100 RP-18, 5 m, $4.0 \times 250$ mm I.D.; Darmstadt, Germany) and by isocratic elution, using $100 \%$ methanol as the mobile phase; the flow rate of the eluent was $1.0 \mathrm{~mL} / \mathrm{min}$. Trichloroacetic acid, chloroform-sulfuric acid and glacial acetic acid-acetyl chloride were used for the qualitative detection of crude triterpenoids as previously described in $\mathrm{Wu}$ (2004).

\section{Hens, management and egg sample collection}

A total of 12022 -week-old laying hens (Hendrix) were randomly allocated into 4 dietary groups each containing 30 birds, including 10 replicates per group and 3 birds per replicate. The birds were housed in individual wire cages $(43 \times 40 \times 60$ $\mathrm{cm}$ ) with a nipple drinker. Over an 8 -week period, each group, including the control group, received a corn-soybean meal diet. The three non-control groups had their diet supplemented with $0.5,1.0$, or $2.0 \%$ dried PESR, respectively. The temperature was set at $25^{\circ} \mathrm{C}$ and relative humidity at $55 \%$. Table 1 presents the ingredients and nutrient compositions of the control diet. Control diet was formulated to meet or exceed the nutrient requirements of laying hens (National Research Council, 1994). The proximate composition was analyzed according to the AOAC (1980). The experimental protocols were approved by the Animal Care and Use Committee of National Chung Hsing University. Feed and water were provided ad libitum, and the light regimen was $16 \mathrm{~h}$ of continuous light per day. Body weights of laying hens were determined at the beginning and end of this study. Eggs were weighed and egg production was daily and individually recorded; the laying rate was calculated 
throughout the experimental period. Feed intake was recorded on a replicate basis at weekly intervals and calculated as $\mathrm{g} /$ day/bird. The value of the feed conversion ratio (FCR) was calculated on a weekly basis for each group. The FCR was expressed as kilograms of feed consumed per kilogram of egg produced. Two eggs were collected randomly in each replicate per group every Sunday to measure the egg quality during the experimental period. Egg weights, egg white weights, egg yolk weights and shell weights were measured. Eggshell strength was evaluated using an eggshell strength tester (DET 6000, NABEL Co., Ltd. Kyoto, Japan). A constantly increasing load was applied to an egg lying lengthways until it broke. The applied load at the time of breakage is the measured strength. The Haugh unit values were calculated using the Haugh unit formula based on the height of albumen determined by an albumen height analyzer (DET 6000, NABEL Co. Ltd., Kyoto, Japan) and egg weight. Eggshell thickness was determined as a mean value of measurements at three locations on the eggs (sharp end, blunt end and middle section) by using a dial pipe gauge. The yolks were separated from the albumen, $100 \mathrm{mg}$ samples of yolks were weighed in the tube, and then yolk lipids were extracted with isopropanol $(5.0 \mathrm{~mL})$, vortexed for $2 \mathrm{~min}$ and centrifuged at $1000 \times \mathrm{g}$ for $15 \mathrm{~min}$. Yolk cholesterol concentrations $(\mathrm{mg}$ of cholesterol/yolk) were determined in the filtered samples by ultraviolet spectrophotometer using commercial kits and calculated according to the method as described in Boehringer Mannheim GmbH Biochemica (1989) and Kaya et al. (2001).

Table 1. Ingredients and nutrient compositions of the control $\operatorname{diet}^{1}$

\begin{tabular}{lc}
\hline \multicolumn{1}{c}{ Item } & Content \\
\hline \multicolumn{1}{c}{1} & 2 \\
\hline Ingredients: & 56.49 \\
corn (\%) & 21.80 \\
soybean meal (44\% of CP) (\%) & 3.20 \\
corn dried distillers grains with solubles (DDGS) (\%) & 3.00 \\
fish meal (\%) & 1.40 \\
wheat bran (\%) & 1.00 \\
full-fat soybean meal (65\% of CP) (\%) & 2.60 \\
tallow (\%) & 7.30 \\
limestone (\%) & 2.60 \\
dicalcium phosphate (\%) & 0.13 \\
DL-Methionine (\%) & 0.15 \\
salt (\%) & 0.08 \\
choline chloride (\%) & 0.25 \\
vitamin-mineral premix ${ }^{2}(\%)$ & 100.0 \\
total (\%) & \\
Chemical-nutritional analysis: & 2810 \\
ME (kcal/kg) & 17.7 \\
crude protein (\%) & 5.7 \\
ether extract $(\%)$ & 4.30 \\
calcium (\%) & 0.50 \\
phosphate (\%) & \\
\hline
\end{tabular}


Table 1 contd.

\begin{tabular}{|c|c|}
\hline . & 2 \\
\hline \multicolumn{2}{|l|}{ PESR chemical composition: } \\
\hline dry matter $(\%)$ & $93.1 \pm 0.3$ \\
\hline crude protein $(\%)$ & $8.6 \pm 0.1$ \\
\hline crude ash (\%) & $5.6 \pm 0.04$ \\
\hline crude fiber $(\%)$ & $13.3 \pm 0.3$ \\
\hline ether extract (\%) & $1.8 \pm 0.02$ \\
\hline calcium $(\%)$ & $0.56 \pm 0.01$ \\
\hline phosphate (\%) & $0.47 \pm 0.01$ \\
\hline
\end{tabular}

${ }^{1}$ The control group was fed the basal diet (corn-soybean meal). Other groups were added with $0.5,1.0$, or $2.0 \%$ of Pleurotus eryngii stalk residue (PESR) in the basal diet, respectively.

${ }^{2}$ Diets contained per kilogram of diet: vitamin A 8,000 IU/kg; vitamin D 1,500 IU/kg; riboflavin $4 \mathrm{mg} / \mathrm{kg}$; cobalamin $10 \mu \mathrm{g} / \mathrm{kg}$; vitamin E $15 \mathrm{mg} / \mathrm{kg}$; vitamin K $2 \mathrm{mg} / \mathrm{kg}$; choline $500 \mathrm{mg} / \mathrm{kg}$; niacin $25 \mathrm{mg} / \mathrm{kg}$; manganese $60 \mathrm{mg} / \mathrm{kg}$; zinc $50 \mathrm{mg} / \mathrm{kg}$; iron $50 \mathrm{mg} / \mathrm{kg}$; copper $3 \mathrm{mg} / \mathrm{kg}$; selenium $0.26 \mathrm{mg} / \mathrm{kg}$

Blood collection for serum cholesterol and antioxidant enzyme determination

Five $\mathrm{mL}$ of blood was also collected at weeks 4 and 8 from the same one layer per replicate (10 birds per group) from their left wing veins using a sterilized syringe and needle for measuring the cholesterol and antioxidant enzyme content. Serum samples were isolated approximately 4 to $5 \mathrm{~h}$ at room temperature after collection, and centrifuged at $3,000 \times \mathrm{g}$ for $30 \mathrm{~min}$ at $4^{\circ} \mathrm{C}$; samples were then stored at $-4^{\circ} \mathrm{C}$ for up to 2 days until analysis. The total cholesterol, high-density lipoprotein cholesterol (HDL), and triglycerides in the serum samples were analyzed with an autoanalyzer (Automatic Biochemical Analyzer, RA-1000; Bayer Corp., Tarrytown, NY) using colorimetric methods and kits purchased from Denka Seiken Co., Ltd. (Tokyo, Japan) for total cholesterol (T-CHO-SN), HDL (HDL-EX) and triglycerides (TGEX) assay kits. A spectrophotometer was used to colorimetrically assay the activities of Trolox equivalent antioxidant capacity (TEAC), CAT and SOD (Wheeler et al., 1990). The procedures were conducted with assay kits purchased from Cayman Chemical Co. Ltd., (Ann Arbor, MI, USA). Serum samples were measured in triplicate and at the appropriate dilutions allowing the enzymatic activities to achieve the linear range of standard curves. Antioxidant enzyme activities were expressed as unit (U) per milliliter of serum.

\section{Statistical analysis}

The data were analyzed by performing ANOVAs for completely randomized designs using the GLM procedure of the SAS software program (1999). Significant statistical differences among the various treatment group means were determined using Duncan's multiple range test. A threshold value of $\mathrm{P}<0.05$ was set to denote statistical significance.

\section{Results}

PESR chemical analysis, active ingredients and layer performance

The chemical composition of dried PESR is shown in Table 1. The PESR contains dry matter $93.1 \pm 0.3 \%$, crude protein $8.6 \pm 0.1 \%$, crude ash $5.6 \pm 0.04 \%$, crude 
fiber $13.3 \pm 0.3 \%$, ether extract $1.8 \pm 0.02 \%$, calcium $0.56 \pm 0.01 \%$ and phosphate $0.47 \pm 0.01 \%$. Moreover, the dried PESR had abundant active ingredients/functional components, including: crude soluble polysaccharide (165.0 mg/g DW), ß-1-3-Dglucan $(0.6 \mathrm{mg} / \mathrm{g} \mathrm{DW})$, adenosine $(58.9 \mu \mathrm{g} / \mathrm{g} \mathrm{DW})$, ergosterol $(1.28 \mathrm{mg} / \mathrm{g} \mathrm{DW})$, total phenolic compounds (5.0 mg gallic acid equivalent (GAE)/g DW) and crude triterpenoids $(1.8 \mathrm{mg} / \mathrm{g}$ DW). Table 2 presents the effect of PESR on performance of laying hens. None of laying hens died, and all appeared to remain healthy throughout the entire period. The feed intake, laying rate, egg mass and feed conversion rate (FCR) were similar among the groups. From 0 to 4 weeks and 5 to 8 weeks, egg mass was approximately 55 and $60 \mathrm{~g} /$ day/bird, and FCR was $1.83-1.88$ vs $1.76-1.84$. As the levels of PESR increased from $0.5 \%$ to $2.0 \%$ of the diet, there was no significant improvement in the hens' performance.

\section{Egg quality and cholesterol content}

Table 3 shows the effect of PESR on egg quality in laying hens. The egg weight, yolk weight, albumen weight, shell weight, shell strength and shell thickness showed no significant difference among the treatment groups. The Haugh unit was significantly higher in the $1.0 \%$ and $2.0 \%$ PESR groups than in the control group. The cholesterol content of the $1.0 \%$ and $2.0 \%$ PESR groups was significantly lower than in the control and $0.5 \%$ PESR groups at 5 to 8 weeks (Table 4 ).

\section{Serum triglycerides and cholesterol content}

Table 5 presents the effect of PESR on serum triglycerides and cholesterol content in laying hens. The serum triglyceride content was lower in the $1.0 \%$ and $2.0 \%$ PESR groups than in the control group, and blood cholesterol at 8 weeks of age between the $1.0 \%$ and $2.0 \%$ PESR groups was significantly lower than in the control group. The HDL content showed no significant difference among the treatments.

\section{Serum antioxidant enzyme activities}

Table 6 shows the effect of PESR on serum antioxidant enzymes in laying hens. The serum antioxidant activities showed higher levels of TEAC, CAT and SOD in the $1.0 \%$ and $2.0 \%$ PESR groups; however, no significant effect was shown between these two groups.

\section{Discussion}

For centuries, mushrooms and their fermented products have been used to maintain human health (Manzi et al., 2004; Lee et al., 2011). Our previous study demonstrated that the PESR still contains abundant secondary metabolites (functional components such as total phenolic compounds, adenosine and ergosterol) that could decrease lipid peroxidation and suggest potential as antioxidants and also as a feed additive (Lee et al., 2012). However, the influence of supplementating hen's diets with PERS and its effect on egg quality characteristics has not yet been evaluated. 


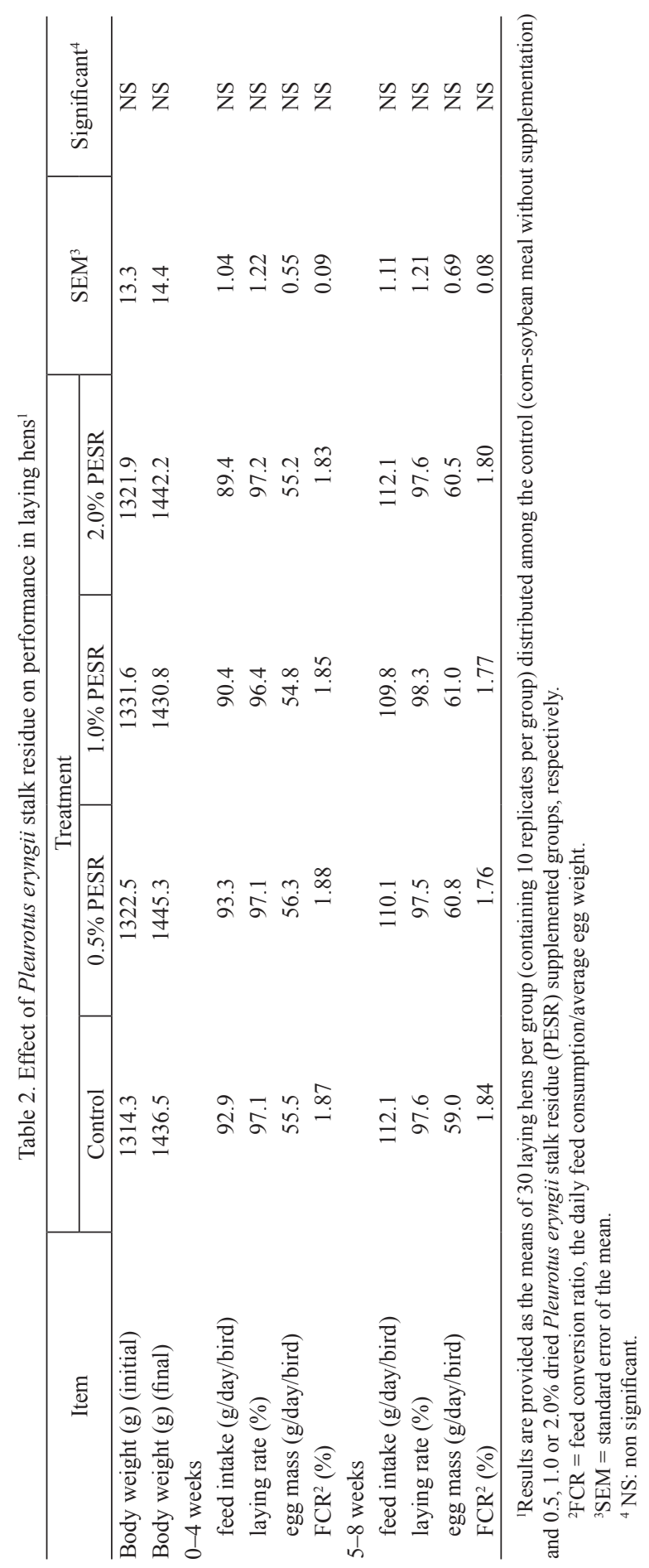




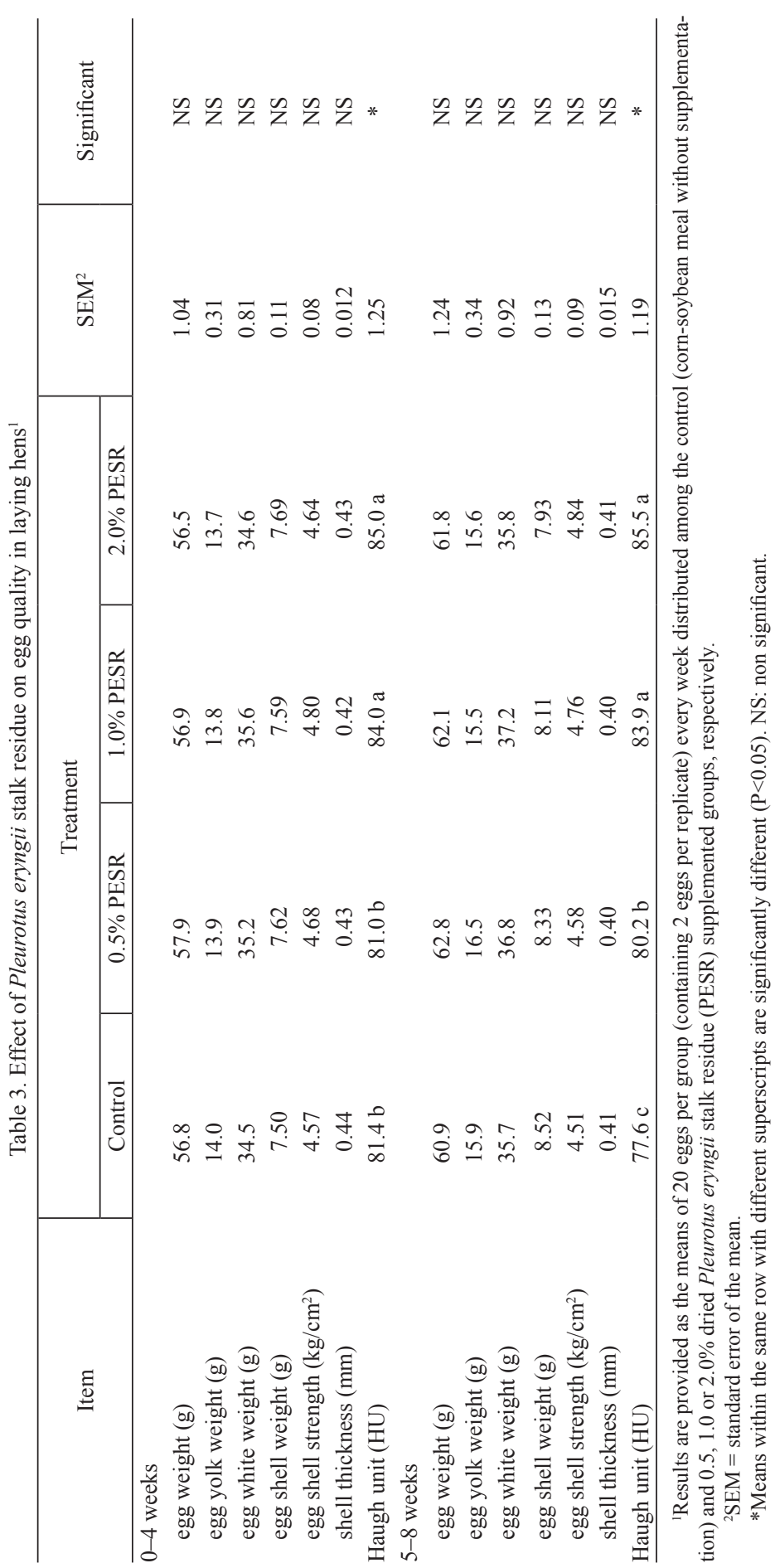




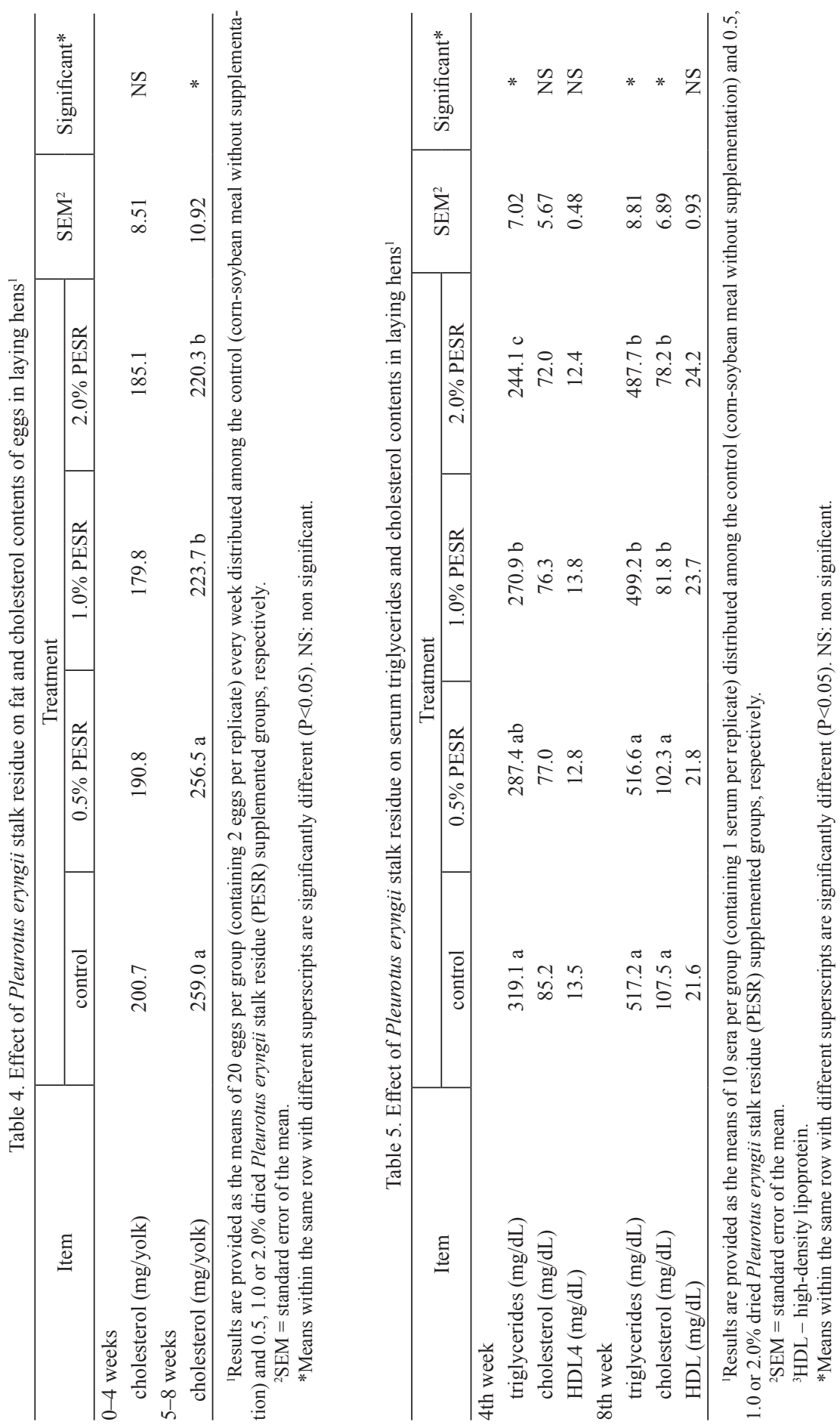




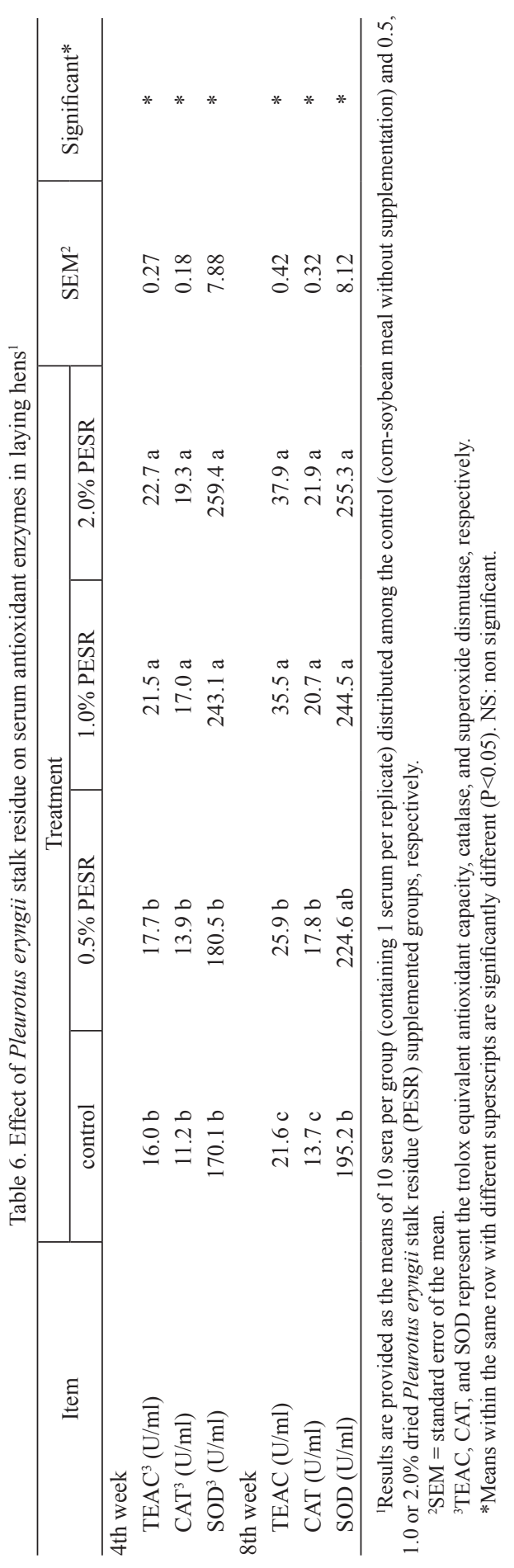


Phenolic compounds, representing some of the major secondary metabolites, are widely found as functional metabolites in mushrooms; their contents could be used as a critical index for determining the antioxidant activity (Liu et al., 2010). The phenolic compounds of dried PESR had approximately 5.0 mg GAE/g DW. Previous studies showed that feed additive, which shows rich antioxidant activities, may increase the fresh value of eggs and result in a higher Haugh unit (Liu et al., 2014; Al-Harthi et al., 2009). In the present study, the Haugh unit scores of $1.0 \%$ and $2.0 \%$ PESR dietary supplemented groups were significantly higher compared to the control group. Liu et al. (2013) reported that adding $0.4 \%$ quercetin, a natural polyphenol compound (flavonoid), could significantly improve the antioxidant capacity and enhance the Haugh unit of eggs in late-laying hens. Olobatoke and Mulugeta (2011) revealed that a $3.0 \%$ addition of dietary garlic powder could markedly increase the Haugh unit of eggs. They also showed that a functional compound such as allicin (an antioxidant in garlic) has beneficial effects for antioxidant activity and hypocholesterolemic effects in hens; however, a larger addition between $3.0 \%$ and $5.0 \%$ had no effect on the Haugh unit score. Similarly, in this study, the $2.0 \%$ addition of PESR seemed to have no significant effect as compared to the $1.0 \%$ supplementation with respect to affecting the Haugh unit.

Choi et al. (2010) showed that dietary antioxidants supplementation, such as with $\alpha$-tocopherol and/or garlic powder, resulted in lower crude fat content in the chicken tissues, suggesting that the activities of hydroxymethylglutaryl-coenzyme A reductase, cholesterol $7 \alpha$-hydroxylase and fatty acid synthetase were markedly reduced. Yeum and Russell (2002) pointed out that diets with the addition of carotenoids (a kind of natural antioxidant) are associated with a reduction of the serum cholesterol content in humans. They explained that carotenoids are absorbed through the intestinal cells as well as incorporated into the chylomicron (triglyceride-rich lipoproteins); then, they enter the circulatory system, forming the chylomicron remnant by the action of lipoprotein hydrolase, and leading to a decrease of triglycerides and cholesterol accumulation in the serum. Salma et al. (2007) reported that adding Rhodobacter capsulatus could decrease triglycerides and cholesterol accumulation in serum and egg yolk. They mentioned that $R$. capsulatus may exert its hypocholesterolemic action through the mechanism of bile acids. Accordingly, $R$. capsulatus may stimulate the binding of cholesterol with bile acids. These functions and the inhibition of micelle formation, combined with the effect of fermentation on short chain fatty acid production, are mechanisms that have been proposed to explain the potential result of lowering serum cholesterol concentration effects (St-Onge et al., 2000). Hence, high levels of fibrous material such as PESR (crude fiber 13.3\%), and garlic husks can mediate increased bile acid excretion and decreased serum/meat tissue lipid levels (Kim et al., 2009). Aydin et al. (2008) showed that diets supplemented with black cumin, a kind of phytogenic, at 20.0 or $30.0 \mathrm{~g} / \mathrm{kg}$ could significantly decrease the egg cholesterol content compared to the group without its addition (control group). These results suggest that our supplementation scheme may affect the lipid synthesis as well as cause decreased cholesterol content accumulation in eggs.

Previously, our results demonstrated the high antioxidant activity of PESR (Lee et al., 2012). The results of serum TEAC values were significantly higher in the 
0.5 to $2.0 \%$ PESR groups compared to the control groups of 35-day-old male broilers. Hence, the antioxidative enzymatic activities of the CAT and SOD displayed the same trend as the TEAC values. In this study, similarly, the serum antioxidant activities showed higher levels of the TEAC, CAT and SOD in the $1.0 \%$ and $2.0 \%$ PESR supplemented compared to $0.5 \%$ PESR supplemented and control groups. Antioxidant enzymes are synthesized and regulated endogenously; they are an important index of the oxidative status of animal tissues (Giannenas et al., 2010). The SOD is an important antioxidant defense system that catalyzes the dismutation of a superoxide anion $\left(\mathrm{O}^{2-}\right)$ into hydrogen peroxide and prevents the generation of free radicals. The CAT converts $\mathrm{H}_{2} \mathrm{O}_{2}$ into $\mathrm{H}_{2} \mathrm{O}$, and the TEAC determines the capacity of a compound to capture ABTS (2,2'-azino-bis-(3-ethylbenzothiazoline-6-sulfonic acid), a radical cation. Brenes et al. (2008) found that the inclusion of $3.0 \%$ grape pomace concentrate increased the oxidative status (TEAC value using the ABTS method) in the serum of broiler chickens; they pointed out that the secondary metabolites found in the aforementioned extracts affected the gene expression of related antioxidant enzymes in animal tissues. Jiang et al. (2007) showed that when soybean isoflavone (ISF, a high-antioxidant product from soybean) was added to the chicken diet, antioxidant enzymatic activities (especially CAT and SOD values) increased in the plasma. Zhao et al. (2011) observed that dietary supplementation of ginger powder $(0.5 \%$ to $2.0 \%)$, a kind of phytogenic, may improve the serum antioxidant status (TEAC and SOD values). Liu et al. (2014) showed that supplementation with quercetin, which is well-known for high antioxidant effects and rich phenolic components, could improve SOD activity in laying hens. The above results demonstrate that dietary supplementation with PESR may raise the oxidative status in laying hens due to the increased serum TEAC, CAT and SOD enzyme activities.

\section{Conclusion}

In conclusion, the results found in the present study imply that PESR, a by-product of fruiting bodies of Pleurotus eryngii, could produce lower cholesterol eggs and raise the oxidative status with $1.0 \%$ and $2.0 \%$ PESR supplementation in laying hens.

\section{References}

A 1 - H a r th i M.A., E 1 - D e e k A.A., A t t i a Y.A., B over a F., Q o t a E.M. (2009). Effect of different dietary levels of mangrove (Laguncularia racemosa) leaves and spice supplementation on productive performance, egg quality, lipid metabolism and metabolic profiles in laying hens. Brit. Poultry Sci., 50: 700-708.

AOAC (1980). Official Methods of Analysis of the Association of Official Analytical Chemists, 13th ed. Association of Official Analytical Chemists, Washington DC.

Ayd in R., Karaman M., C i c e k T., Yardibi H. (2008). Black cumin (Nigella sativa L.) supplementation into the diet of the laying hen positively influences egg yield parameters, shell quality, and decreases egg cholesterol. Poultry Sci., 87: 2590-2595.

Boehringer Mannheim GmbH. Biochemica (1989). Methods of Biochemical Analysis and Food Analysis. Boehringer Mannheim GmbH Biocemica, Mannheim, Germany, pp 26-28.

Brenes A., Viveros A., Goñi I., C enten o C., S áy a g o-Ayerdy S.G., Arija I., S a u ra - Calixt o F. (2008). Effect of grape pomace concentrate and vitamin E on digestibility of polyphenols and antioxidant activity in chickens. Poultry Sci., 87: 307-316. 
Choi I.H., P a rk W.Y., K i m Y.J. (2010). Effects of dietary garlic powder and $\alpha$-tocopherol supplementation on performance, serum cholesterol levels, and meat quality of chicken. Poultry Sci., 89: 1724-1731.

Giannen as I., Pappas I.S., Mavridis S., Kontopid is G., Skoufos J., Kyriazakis I. (2010). Performance and antioxidant status of broiler chickens supplemented with dried mushrooms (Agaricus bisporus) in their diet. Poultry Sci., 89: 303-311.

Ji ang Z.Y., Jiang S.Q., L in Y.C., Xi P.B., Yu D.Q., Wu T.X. (2007). Effects of soybean isoflavone on growth performance, meat quality, and antioxidation in male broilers. Poultry Sci., 86: $1356-1362$

K a y a S., Keçe ci T., Hali lođlu S. (2001). Effects of zinc and vitamin A supplements on plasma levels of thyroid hormones, cholesterol, glucose and egg yolk cholesterol of laying hens. Res. Vet. Sci., 71: 135-139.

K i m Y.J., J in S.K., Y a n g H.S. (2009). Effect of dietary garlic bulb and husk on the physicochemical properties of chicken meat. Poultry Sci., 88: 398-405.

Kuj al a T.S., L op on en J.M., K lik a K.D., P ihla j a K. (2000). Phenolics and betacyanins in red beetroot (Beta vulgaris) root: distribution and effect of cold storage on the content of total phenolics and three individual compounds. J. Agric. Food Chem., 48: 5338-5342.

Lee T.T., Tian Y.R., Yu B. (2011). Polysaccharide, adenosine and crude triterpenoid contents of Pleurotus eryngii and its immunostimulant capacity in vivo. J. Agric. Sci. Technol., A1: $1166-1169$.

Lee T.T., Ciou J.Y., Chiang C.J., Cha o Y.P., Yu B. (2012). Effect of Pleurotus eryngii stalk residue on the oxidative status and meat quality of broiler chickens. J. Agric. Food Chem., 60: $11157-11163$.

L e e T.T., C i o u J.Y., C h e n C.L., Yu B. (2013). Effect of Echinacea purpurea L. on oxidative status and meat quality in Arbor Acres broilers. J. Sci. Food Agric., 93: 166-172.

L in H., Decuypere E., B u y s e J. (2006). Acute heat stress induces oxidative stress in broiler chickens. Comp. Biochem. Physiol. A Mol. Integr. Physiol., 144: 11-17.

L i u H.N., Li u Y., H u L.L., S u o Y.L., Zhang L., J in F., Feng X.A., Ten g N., L i Y. (2014). Effects of dietary supplementation of quercetin on performance, egg quality, cecal microflora populations, and antioxidant status in laying hens. Poultry Sci., 93: 347-353.

L i u Y., Li Y., Li u H.N., S u o Y.L., H u L.L., F eng X.A., Zh ang L., J in F. (2013). Effects of quercetin on performance and egg quality during the late laying period of hens. Brit. Poultry Sci., 54: $510-514$.

Li u X., Zhou B., Lin R., Ji a L., D eng P., F an K., Wang G., Wang L., Zhang J. (2010). Extraction and antioxidant activities of intracellular polysaccharide from Pleurotus sp. mycelium. Int. J. Biol. Macromol., 47: 116-119.

Manzi P., Gambelli L., Marconi S., Vivanti V., Pizzoferrato L. (1999). Nutrients in edible mushrooms: an inter-species comparative study. Food Chem., 65: 477-482.

M a n zi P., M ar c o n i S., A g u z z i A. (2004). Commercial mushrooms: nutritional quality and effect of cooking. Food Chem., 84: 201-206.

Ma u J.L., Chang C.N., H u ang S.J., Chen C.C. (2004). Antioxidant properties of methanolic extracts from Grifola frondosa, Morchella esculenta and Termitomyces albuminosus mycelia. Food Chem., 87: 111-118.

Mc Cleary B.V., Glennie-Holmes M. (1985). Enzymic quantification of (1-3)(1-4)- $\beta$-D-glucan in barley and malt. J. Inst. Breew., 91: 285-295.

Mujahid A., Akiba Y., Toyomizu M. (2007). Acute heat stress induces oxidative stress and decreases adaptation in young White Leghorn cockerels by down regulation of avian uncoupling protein. Poultry Sci., 86: 364-371.

Olobat oke R.Y., Mulugeta S.D. (2011). Effect of dietary garlic powder on layer performance, fecal bacterial load, and egg quality. Poultry Sci., 90: 665-670.

S a f a r y H., D a n e s h y a r M. (2012). Effect of dietary sodium nitrate consumption on egg production, egg quality characteristics and some blood indices in native hens of west Azarbaijan province. Asian-Aust. J. Anim. Sci., 25: 1611-1616.

S a lma U., Miah A.G., Tareq K.M.A., Maki T., Ts uji i H. (2007). Effect of dietary Rhodobacter capsulatus on egg-yolk cholesterol and laying hen performance. Poultry Sci., 86: 714-719. 
Seven I., A ksu T., S even P.T. (2010). The effects of propolis on biochemical parameters and activity of antioxidant enzymes in broilers exposed to lead-induced oxidative stress. Asian-Aust. J. Anim. Sci., 23: 1482-1489.

S t - O n g e M.P., F a rnw or th E.R., J on es P.J.H. (2000). Consumption of fermented and nonfermented dairy products: Effects on cholesterol concentrations and metabolism. Am. J. Clin. Nutr., 71: 674-681.

Wh e e le r C.R., S a 1 z m a n J.A., E 1 s a y e d N.M., O m a y e S.T., J r K or t e D.W. (1990). Automated assays for superoxide dismutase, catalase, glutathione peroxidase, and glutathione reductase activity. Anal. Biochem., 184: 193-199.

W u L.J. (2004). Natural Pharmaceutical Chemistry. People's Medical Publishing House, Beijing, pp. 376-377.

Y e u m K.J., R u s s e 11 R.M. (2002). Carotenoid bioavailability and bioconversion. Annu. Rev. Nutr., 22: 483-504.

Z h a o X., Yang Z.B., Yang W.R., Wang Y., Ji ang S.Z., Z h ang G.G. (2011). Effects of ginger root (Zingiber officinale) on laying performance and antioxidant status of laying hens and on dietary oxidation stability. Poultry Sci., 90: 1720-1727.

Received: 3 VI 2014

Accepted: 12 VIII 2014 